\title{
A Literature Review of Personalized Learning Algorithm
}

\author{
Yin Tang, Wen Wang \\ Management School, Jinan University, Guangzhou, China \\ Email: wangwenfuture@163.com
}

How to cite this paper: Tang, Y. and Wang, W. (2018) A Literature Review of Personalized Learning Algorithm. Open Journal of Social Sciences, 6, 119-127. https://doi.org/10.4236/jss.2018.61009

Received: December 19, 2017

Accepted: January 19, 2018

Published: January 22, 2018

Copyright (C) 2018 by authors and Scientific Research Publishing Inc. This work is licensed under the Creative Commons Attribution International License (CC BY 4.0).

http://creativecommons.org/licenses/by/4.0/

\begin{abstract}
With the rapid development of the online learning platform, the learning resources are also unprecedentedly rich. The study of personalized learning algorithms meets the need to provide students with the most suitable resources for learning. This paper constitutes a literature review on personalized learning algorithm at home and abroad. The review aims at elaborating the research based on recommendation system and data mining in personalized learning algorithm and look forward to the future research trend.
\end{abstract}

\section{Keywords}

Personalized Learning, Data Mining, Recommendation System

\section{Introduction}

Students' differences in individual differences, interest and style of learning have a great influence on the effect of learning. The "student-centered" personalized learning emphasizes students' interest, learning styles, differences in cognitive level and other aspects as the basis, to provide the most suitable learning resources and their teaching design for each student, and strive to improve the learning efficiency, truly individualized. With the development of modern society, many diverse learning resources are also easy for online learners to generate cognitive overload and Internet addiction. People's voice for personalized learning is increasing. The advancement of educational informatization has made the personalized learning algorithm develop rapidly. Currently, there are mainly two aspects of personalized learning algorithm research: one is based on recommendation system, and the other is based on data mining method. This paper collates the development process and effect of personalized learning algorithm based on recommender system and data mining method, and puts for- 
ward the possibility of application of reinforcement learning in personalized learning algorithm.

\section{Personalized Learning Algorithm Based on Recommendation System}

Traditional search or recommendation methods can only provide the same search results for all users but they are unable to make personalized learning recommendations. Therefore, the personalized recommendation research was proposed in the 1990s [1] [2]. At present, the personalized learning recommendation algorithm proposed by scholars at home and abroad mainly includes content-based recommendation algorithm, collaborative filtering recommendation algorithm and hybrid recommendation algorithm.

\subsection{Content-Based Recommendation Algorithm}

The content-based recommendation algorithm is relatively simple and intuitive. It is based on the user's historical interest information to recommend the resources that may be of interest to the user. By analyzing the recent navigation history of learners, Mohamed et al. [3] recommend the learning resources. In combination with the need of learning, Chen Min et al. [4] propose a personalized recommendation model for ubiquitous learning. With the core of ontology technology, Jiang Qiang et al. [5] propose a personalized ontology learning resource recommendation system based on the user model. Khribi et al. [6] make a similar match between user's recent navigation history and learning resources, which automatically generates learning suggestions online. Sharif et al. [7] design a recommended framework, through the key learning resources and the user's interest in learning the label match to implementation of the recommendations, and assign weights learning resources, learning resources will be further sorted according to the importance level.

The content-based recommendation algorithm only considers the matching of learning resources and users' interest characteristics without considering the similarity between users, which results in only recommending resources that users have learned and are not interested in [8]. But there is no recommendation for learning resources that are not available to users. In order to avoid the disadvantages of the content-based learning recommendation, the researchers propose a new personalized solution, for example, the collaborative filtering recommendation technology [9].

\subsection{Collaborative Filtering Recommendation Algorithm}

According to different users' preference for the same learning content, the user-based collaborative filtering recommendation computes the distance between users and then recommends learning content between users with the same preference. Based on analyzing the three Service modes of cloud computing, Wang Ping et al. [10] propose a learning resource recommendation system based on 
the PAAS (Platform as a Service) model. Liu Xiaoxue [11] proposes an adaptive recommendation system based on current learners' online behaviors which automatically recommend learning resources online. Wang Honglei [12] puts forward a similar learning partner recommendation system based on social tagging in response to the "information overload" and "information trek" problems faced by learners in Internet learning. Xu Tianwei et al. [13] propose a collaborative filtering algorithm based on the project, user and attribute value matrix for the lack of personalized curriculum recommendation and efficiency of elective courses in university selection system. Wang Yonggu et al. [14] try to introduce the latest research results in the e-commerce field into education, and propose a collaborative filtering learning resource recommendation system. The collaborative filtering can provide recommendations with less feedback and is more efficient, but there are two obvious defects for it [15]. The primary defect is the sparsity problem. Many users are reluctant to evaluate the learning resources, which results in the lack of basic data of the user similarity calculation, thus affects the recommendation accuracy. For the data sparse problem of collaborative filtering algorithm, by introducing social tagging, Li Ning et al. [16] recommend the learning resources of interest to the learners. The second is the cold-starting problem. Many newly launched learning resources are rarely recommended even if learning resources are valuable because of the lack of evaluation data. Based on the properties of learning resources in multiple spaces, Saleh et al. [17] propose the tree model to model the interest of the users, and propose the new similarity calculation method in the scholar tree model. The experimental results show that the proposed method can effectively alleviate the problem of cold-starting and sparsity.

\subsection{Hybrid Recommendation Algorithm}

To overcome the shortcomings of content-based recommendation and collaborative filtering recommendation, most scholars try to combine these two modes in some way to recommend. Some scholars try to re-enter the results of the content-based recommendation to the collaborative filtering recommendation system, and adopt the collaborative filtering recommendation technology for secondary filtering. Some scholars try to re-enter the results of the collaborative filtering recommendation to the content-based recommendation [18]. Ge et al. [19] propose a recommendation that combines the content-based recommendation and collaborative filtering recommendation. Mou Zhijia et al. [20] analyze the personality characteristics of students and put forward personalized recommendation system based on electronic schoolbag which is based on the establishment of learner model. From the perspective of learning support services of virtual learning community, Yang Lina et al. [21] study the personalized recommendation of learning resources for learners with different working backgrounds and learning needs.

Because the recommend ideas of content-based recommendation and colla- 
borative filtering recommendation are different, how to combine the two effectively is the core content of hybrid recommendation. Although many scholars have proposed mixed methods, the accuracy and efficiency of hybrid recommendation is worth further improvement.

\section{Personalized Learning Based on Data Mining}

Data mining is used to extract hidden information in user's learning behavior data for knowledge discovery. It has two tasks: one is to describe; the other is to predict. For the former, it is mainly to according to the content of the data in the behavior database, through the right way to make statements on the data, data classification, clustering, association rules mining, so as to find the hidden rules between the data. The latter is based on existing data in the behavior database to predict or infer the future, such as, sequential pattern mining, genetic algorithm and swarm intelligence algorithm. In personalized learning algorithm, the methods of data mining mainly include classification algorithm, clustering algorithm, association rule mining, sequence pattern mining algorithm, genetic algorithm and swarm intelligence algorithm.

\subsection{Classification Algorithm}

The classification algorithm is mainly used to extract the characteristics of various learners and individualize education to them. Feldman et al. [22] use the number of attempts, duration, and final grades of students in a game to train naive Bayes classifier to determine the student's learning style. Damez et al. [23] use the method of fuzzy decision tree to model students, by analyzing the cognitive characteristics of students' interaction with learning system, and differentiating between online learning experience and novice. Natek et al. [24] use the decision tree algorithm to classify the students to get the characteristics of personal information and teaching links of students with different grades. The analysis finds that the factors that affect the final score of the course have the learning type, the activity performance in the learning process, daily test scores and so on. Chanchary et al. [25] use the association rule mining and decision tree classification method to find out the relationship between the user's use of learning management system and the final grade.

\subsection{Clustering Algorithm}

The clustering algorithm can be used to analyze the isolated point and detect the abnormal learning behavior and fraud behavior of the learners, to remind the teachers to deal with it. In addition, clustering algorithm can also be a preprocessing step for other mining methods. The results of clustering algorithm are used for further data mining analysis, and the more profound and unknown characteristics of each type of learner are obtained to improve its accuracy and efficiency. Or to compare the results of further analysis, differences among different groups are found out. Ayers et al. [26] use hierarchical clustering, K-means 
algorithm, and model-based clustering algorithm to perform cognitive diagnostics. Milos et al. [27] use K-means algorithm to divide students into three categories according to students' behaviors and analyze the cognitive characteristics of various students by combining the information of students' cognitive style collected by the MBTI scale. Araya et al. [28] analyze the data of a large multiplayer online math game and discovery the rules of teamwork. Teng et al. [29] cluster the learners, analyze the clustering results and provide specific suggestions for each class, based on the similarity of learning behavior. Li Shuang et al. [30] use the clustering method to divide learners into five categories based on their behavioral sequence characteristics. By analyzing the behavioral sequence and behavioral transformation patterns generated by online learning of various learners, they define these five categories as low input, shallow input, performance input, progressive and random participation.

\subsection{Association Rules Mining and Sequential Pattern Mining Algorithm}

Association rules and sequential pattern mining are applied to discover the learning and usage habits of learners' online learning. For example, learning activities should be arranged to implement personalized learning recommendations and learning arrangements. For example, Yu et al. [31] use online time, the number of reading documents, and the number of questions to describe the behavior of learners. The relationship between each learning behavior model is explored by using fuzzy association rule mining. Wu Qin et al. [32] use the Kolb learning style scale to obtain the student's learning style, and use the association rule mining method to obtain the behavioral characteristics of various learning styles. Chen et al. [33] use association rule mining to find out that students' misunderstanding of certain knowledge points which will lead to misunderstandings of other specific knowledge points. Aher et al. [34] apply Apriori algorithm to analyze the association rules of the course learning records of all kinds of students based on the K-means algorithm clustering to get all kinds of students' preferred courses to recommend suitable courses to students. Wu Shuping [35] uses the sequence pattern mining algorithm to unify the learning behavior of learners to obtain the learning path network diagram, and analyze the high frequency learning path found the effective learning mode. Pahl et al. [36] use the sequential pattern mining method to obtain the learning path of online learners, and compare it with the teacher's expected path, and then find the deviation from the expectation of timely intervention.

\subsection{Genetic Algorithm and Swarm Intelligence Algorithm}

Wang et al. [37] implement a self-adaptive learning system by using BP neural network method, which can be used to recommend different learning materials based on the learners' gender, personality and degree of learning anxiety. Xiao Huimin et al. [38] first feature the learners, based on the particle swarm algo- 
rithm, then make each learner as a particle and make the path selection in the learning process and evaluation value as a representative of the its space for personalized learning path optimization by using Particle Swarm Optimization (PSO) algorithm. Ahmad et al. [39] introduce the idea of using ant colony optimization algorithm and concept graph to recommend suitable learning paths for different learning groups. Cheng Yan [40] proposes the extension and colony algorithm to solve the recommendation problem of learning path. The evaluation of learning path and the characteristics of target users in knowledge level and learning style are comprehensively referenced in the recommendation decision.

\section{Conclusion}

Our target in this paper is to present a literature review of personalized learning algorithm research from two aspects of the recommendation system and data mining. Through the statistical analysis of the current individualized learning algorithm, the different approaches that are applied to construct them are compared. It can be obtained that recommendation system ignores the information contained in user behavior. Using data mining for user behavior helps us understand the regularity of user's use of the system, but there are certain defects. For example, the associated rules technology is vulnerable to the loss of important patterns, which leads users to not be interested in the mode of mining and the application of sequential pattern to personalized recommendation coverage is low. However, for the recommendation system based on data mining algorithm, its efficiency depends on which data mining algorithm is adopted. The system is often faced with low resource utilization, unstable recommendation output and low accuracy. At present, Reinforcement Learning (RL) is an important method to solve Sequential Decision Making, which is closely combined with deep Learning in the field of artificial intelligence, has achieved remarkable results, and becomes the current breakthrough cognitive intelligence representative machine learning method. Li Yiqun et al. [41] propose a label recommendation algorithm, based on the framework of reinforcement learning theory. The sparse data is constructed through simulated user interest, combining simulated data with historical user access data for collaborative filtering recommendations. Therefore, it seems that the reinforcement learning has a good feasibility in the learner modeling and the learning sequence recommendation. So, how to start from reinforcement learning, build learner models, model online learning behavior, and provide learners with their own learning strategies automatically and dynamically will become a research trend in the future.

\section{References}

[1] Resnick, P., Iacovou, N., Suchak, M., et al. (1994) GroupLens: An Open Architecture for Collaborative Filtering of Netnews. Proceedings of the 1994 ACM Conference on Computer Supported Cooperative Work, Chapel Hill, NC, 22-26 October 1994, 175-186. https://doi.org/10.1145/192844.192905

[2] Hill, W., Stead, L., Rosenstein, M., et al. (1995) Recommending and Evaluating 
Choices in a Virtual Community of Use. Proceedings of the SIGCHI Conference on Human Factors in Computing Systems, Denver, CO, 7-11 May 1995, 194-201. https://doi.org/10.1145/223904.223929

[3] Mohammadiari, S. and Singh, H. (2015) Understanding the Effect of E-Learning on Individual Performance: The Role of Digital Literacy. Computer \& Education, 82, 11-25. https://doi.org/10.1016/j.compedu.2014.10.025

[4] Chen, M., Yu, S.Q., Yang, X.M., et al. (2011) The Design of Personalized Recommendation Model for the Content of the Study. Modern education technology, 21, 13-18.

[5] Jiang, Q., Zhao, W., Du, X., et al. (2010) The Personalized Ontology Learning Resources Recommended Study. China Audio-Visual Education, 5, 106-111.

[6] Khribi, M.K., Jemni, M. and Nasraoui, O. (2009) Automatic Recommendations for E-Learning Personalization Based on Web Usage Mining Techniques and Information Retrieval. Educational Technology and Society, 12, 30-42.

[7] Sharif, N., Afzal, M.T. and Helic, D. (2012) A Framework for Resource Recommendations for Learners Using Social Bookmarking. Proceedings of the 8th International Conference on Computing and Networking Technology, IEEE, Gyeongju, 27-29 August 2012, 71-76.

[8] Salehi, M., Kamalabadi, I.N. and Ghoushchi, M.B.G. (2014) Personalized Recommendation of Learning Material Using Sequential Pattern Mining and Attribute Based Collaborative Filtering. Education and Information Technologies, 19, 713-735. https://doi.org/10.1007/s10639-012-9245-5

[9] Salehi, M. and Kamalabadi, I.N. (2013) Hybrid Recommendation Approach for Learning Material Based on Sequential Pattern of the Accessed Material and the Learner's Preference Tree. Knowledge-Based Systems, 48, 57-69. https://doi.org/10.1016/j.knosys.2013.04.012

[10] Wang, P. and Liu, L. (2013) Learning Recommendation System Based on the Pattern of PaaS Cloud Study. Journal of education informationization in China, 3, 78-81.

[11] Liu, X.X. (2012) Based on the Web Use Mining of e-Learning Adaptive Recommend System. Journal of Electrochemical Education Research, No. 4, 82.

[12] Wang, H.L. (2012) Similar Study Partner Recommendation System Based on Social Label Design. Journal of Education Technical Equipment in China, No. 36, 45-48.

[13] Xu, T.W., Song, Y.T. and Duan, C.J. (2014) Study at the Personalized Recommendation System Based on Collaborative Filtering. Journal of Modern Education Technology, 24, 92-98.

[14] Wang, Y.G., Qiu, F.Y., Zhao, J.G., et al. (2011) Based on the Collaborative Filtering Technology of Learning Resource Personalized Recommendation Research. Journal of Distance Education, No. 3, 66-71.

[15] Aher, S.B. and Lobo, L. (2012) Applicability of Data Mining Algorithms for Recommendation System in E-Learning. Proceedings of the International Conference on Advances in Computing, Communications and Informatics, Chennai, 3-5 August 2012, 1034-1040. https://doi.org/10.1145/2345396.2345562

[16] Li, N., Liu, Z.Q. and Wang, Y.B. (2014) Personalized Recommendation on the Learning Resources of Research and Design. Journal of Education Informationization in China, No. 19, 44-47.

[17] Salehi, M. and Kamalabadi, I.N. (2012) Attribute-Based Recommender System for Learning Resource by Learner Preference Tree. Proceedings of the 2 nd Internation- 
al e-Conference on Computer and Knowledge Engineering, Mashhad, 18-19 October 2012, 133-138.

[18] Wan, L. and Zhao, C. (2012) A Hybrid Learning Object Recommendation Algorithm in E-Learning Context. International Journal of Digital Content Technology and Its Applications, 6, 442-448. https://doi.org/10.4156/jdcta.vol6.issue18.53

[19] Ge, L., Kong, W. and Luo, J. (2006) Courseware Recommendation in E-Learning System. Proceedings of the 5th International Conference on Advances in Web Based Learning, Penang, 19-21 July 2006, 10-24.

[20] Mou, Z.J. and Wu, F.T. (2015) Based on the Learner Model in Electronic Schoolbag Personalized Learning Resources Recommended Study. Journal of Electrochemical Education Research, No. 1, 69-69.

[21] Yang, L.N., Liu, K.C. and Yan, Z.J. (2010) Virtual Learning Community Oriented Learning Resource Personalized Recommendation Research. Journal of $\mathrm{Au}$ dio- Visual Education Research, No. 4, 67-67.

[22] Feldman, J., Monteserin, A. and Amandi, A. (2014) Detecting Students Perception Style by using Games. Computers \& Education, 71, 14-22. https://doi.org/10.1016/j.compedu.2013.09.007

[23] Damez, M., Bouchon-Meunier, B., Ha, T., et al. (2005) Fuzzy Decision Tree for User Modeling from Human-Computer Interactions. Proc of the 5 th International Conference on Human System Learning, Marrakech, November 2005, 287-302.

[24] Natek, S. and Zwilling, M. (2014) Student Data Mining Solution-Knowledge Management System Related to Higher Education Institutions. Expert Systems with Applications, 41, 6400-6407. https://doi.org/10.1016/j.eswa.2014.04.024

[25] Chanchary, F.H., Haque, I. and Khalid, M.S. (2008) Web Usage Mining to Evaluate the Transfer of Learning in a Web-Based Learning Environment: International Workshop on Knowledge Discovery and Data Mining. Proc of International Workshop on Knowledge Discovery and Data Mining, Adelaide, 23-24 January 2008, 249-253.

[26] Ayers, E., Nugent, R. and Dean, N. (2009) A Comparison of Student Skill Knowledge Estimates. Proc of International Conference on Educational Data Mining, Cordóba, 1-3 July 2009, 1-10.

[27] Jovanovic, M., Vukicevic, M., Milovanovic, M., et al. (2012) Using Data Mining on Student Behavior and Cognitive Style Data for Improving E-Learning Systems: A Case Study. International Journal of Computational Intelligence Systems, 5, 597-610. https://doi.org/10.1080/18756891.2012.696923

[28] Araya, R., Jiménez, A., Bahamondez, M., et al. (2014) Teaching Modeling Skills using a Massively Multiplayer Online Mathematics Game. World Wide Web-Internet \& Web Information Systems, 17, 213-227.

[29] Teng, B.C., Lin, C., Cheng, S., et al. (2004) Analyzing User Behavior Distribution on E-Learning Platform with Techniques of Clustering. Proc of Society for Information Technology \& Teacher Education International Conference, Atlanta, 1-6 March 2004, 3052-3058.

[30] Li, S., Zhong, Y., Yu, C., et al. (2017) Based on the Analysis of Behavioral Sequence Analysis on Online Learning Participation Model. China Electrochemical Education, No. 3, 88-95.

[31] Yu, P., Own, C. and Lin, L. (2001) On Learning Behavior Analysis of Web Based Interactive Environment. Proc of Implementing Curricular Change in Engineering Education, Oslo/Bergen, 6-10 August 2001, 1-10. 
[32] Wu, Q., Luo, R.G. and Wang, Q.Y. (2015) Empirical Research on Online Learning Behavior Based on Association Rules. Modern Education Technology, 25, 88-94.

[33] Chen, C.M., Hsieh, Y.L. and Hsu, S.H. (2007) Mining Learner Profile Utilizing Association Rule for Web-Based Learning Diagnosis. Expert Systems with Applications, 33, 6-22. https://doi.org/10.1016/j.eswa.2006.04.025

[34] Aher, S.B. and Lobo, L.M.R.J. (2013) Combination of Machine Learning Algorithms for Recommendation of Courses in E-Learning System Based on Historical Data. Knowledge-Based Systems, 51, 1-14. https://doi.org/10.1016/j.knosys.2013.04.015

[35] Wu, S.P. (2013) Analysis and Study of Teacher Network Learning Based on Data Mining. Teacher Education Research, 25, 49-57.

[36] Pahl, C. and Donnellan, D. (2003) Data Mining Technology for the Evaluation of Web-Based Teaching and Learning Systems. Proc of Congress E-Learning, Montreal, 15-19 October 2002, 1-7.

[37] Wang, Y.H. and Liao, H.C. (2011) Data Mining for Adaptive Learning in a TESL-Based E-Learning System. Expert Systems with Applications, 38, 6480-6485. https://doi.org/10.1016/j.eswa.2010.11.098

[38] Xiao, H.M. and Ma, C.J. (2013) The Method of Learning Path Recommendation Based on Particle Swarm Algorithm. Henan Science, No. 12, 2190-2193.

[39] Ahmad, K., Maryam, B.I. and Molood, A.E. (2013) A Novel Adaptive Learning Path Method. 4 th International Conference on E-Learning and E-Teaching, Shiraz, 13-14 February 2013, 20-25.

[40] Cheng, Y. (2011) The Method of Learning Path Recommendation Based on Group Intelligence in Online Learning. Journal of System Management, No. 2, 232-237.

[41] Li, Y.Q., Zhang, W.S., Yang, L. and Liu, Y.Q. (2010) Research and Application of Enhanced Learning Recommendation Algorithm Based on Tag. Computer Application Research, 27, 2845-2847 + 2852 . 